\title{
Pelvic pain following microwave endometrial ablation-is hysteroscopic adhesiolysis worthwhile?
}

\author{
J. A. Allen • K. Phillips
}

Received: 2 April 2008 / Accepted: 9 June 2008 / Published online: 25 June 2008

(C) Springer-Verlag 2008

\begin{abstract}
A proportion of patients present with chronic pelvic pain following microwave endometrial ablation, presumed to be due to the development of a haematometra. This retrospective observational study of 20 patients was performed to determine if hysteroscopy and adhesiolysis is beneficial in this group of patients. Seventeen patients were found to have intrauterine adhesions at hysteroscopy that were divided. At follow-up, nine patients were cured of pain and discharged. Of the remaining 11 patients, one patient with endometriosis underwent laparoscopic bilateral salpingo-oophorectomy. Nine patients underwent hysterectomy and one patient was put on mefenamic acid. In conclusion, some patients were still symptomatic following hysteroscopy and needed further intervention in the form of major gynaecological surgery. However, $45 \%$ of patients improved following only the minor day case procedure, without needing to resort to a hysterectomy with its inherent risks and costs.
\end{abstract}

Keywords Haematometra · Microwave endometrial ablation · Adhesiolysis · Hysteroscopy $\cdot$ Pelvic pain

\section{Introduction}

Microwave endometrial ablation (MEA) is a second generation endometrial ablation technique that is commonly used as a treatment for dysfunctional uterine bleeding. It has been granted National Institute of Clinical Excellence

J. A. Allen $(\bowtie) \cdot K$. Phillips

Castle Hill Hospital,

Castle Road,

Cottingham, North Humberside HU16 5JQ, UK

e-mail: jane.allen@hey.nhs.uk approval and has been shown to be effective in $70-80 \%$ of women [1]. The overall risk of complications associated with MEA is small $[1,2]$, although long term data on complications and success rates is lacking. One complication that has been associated with first generation endometrial ablative techniques is postoperative pelvic pain. This usually presents a few months following surgery with an incidence between $4.7-13.5 \%$ [3-5]. The majority of these women eventually undergo a hysterectomy. Information regarding the incidence and management of this complication following MEA is limited; therefore, this study was devised to assess two main aims. The first was to determine the incidence of pelvic pain post MEA to help facilitate future preoperative counselling of women considering the procedure. The second was to assess if hysteroscopy with adhesiolysis was beneficial in this group of patients by curing pelvic pain, possibly by prevention of a haematometra and whether, as a consequence, hysterectomy or major surgery could be avoided in this group of patients.

\section{Materials and method}

This was a retrospective, observational study of patients who underwent hysteroscopic examination due to a history of pelvic pain following MEA. The patients had all presented to a gynaecological clinic in a UK teaching hospital, due to a history suggestive of intrauterine adhesions, with either cyclical or intermittent crampy pelvic pain. As these patients were thought to have a high risk of intrauterine adhesions from their previous MEA, all hysteroscopic examinations were performed under general anaesthesia in case any complications were encountered. If any intrauterine adhesions were visualised at hysteroscopy, these were divided by blunt dissection with the tip of the 
hysteroscope under direct vision. In a small number of cases, laparoscopic examination was also performed if deemed clinically necessary.

Patients who had a hysteroscopy under general anaesthesia were identified from theatre record books. The case notes were reviewed to ensure the patient had previously had a MEA; if so, then the relevant information was collected. This included the patient's age, previous history of female sterilisation, presence of pain prior to the MEA procedure, history of endometritis post MEA, time from MEA procedure to re-presentation at the gynaecology clinic, type and nature of pain, preoperative ultrasound findings if performed, hysteroscopic findings, the laparoscopic findings, if undertaken and reason for performing laparoscopy, patient satisfaction at follow-up and, if necessary, any further management that was instigated.

\section{Results}

Twenty women aged between 34 and 51 years, with a mean age of 42, were identified from the theatre record books. All hysteroscopic examinations were performed between October 2003 and May 2006, and all MEA procedures were performed between December 2000 and June 2005. The total number of MEA procedures performed in the second time period was 182 , making the incidence of pelvic pain post MEA $11 \%$.

Prior to the MEA procedure, 35\% (7/20) of patients had been sterilised and $60 \%(12 / 20)$ gave a history of pain prior to the MEA procedure. Eight patients described the pain as cyclical and four as non-cyclical. Seventy-five percent $(15 / 20)$ of patients had a pelvic ultrasound performed prior to the hysteroscopy, but only six of these showed evidence of a central haematometra which was then confirmed hysteroscopically in all cases. Four patients gave a history of endometritis post MEA for which they received a course of antibiotics from their General Physician; in the remaining patients, only one denied any history of endometritis as this was not routinely enquired about at the follow-up visit.

The mean time to presentation with pain at the gynaecology clinic following MEA was 16 months with a range of 3-41 months. Nineteen patients presented with a history of pain alone which was cyclical in 13 and intermittent in six. One patient presented with a history of both cyclical pelvic pain and heavy vaginal bleeding.

At hysteroscopy, 85\% (17/20) of patients were found to have intrauterine adhesions that were divided. In two patients, small uterine perforations occurred at the time of hysteroscopy, but as these were done under direct vision, no further treatment was needed and no further complications occurred.
Five patients underwent laparoscopy at the same time as the hysteroscopy. One was the patient presenting with pain and bleeding whom had a repeat MEA done under laparoscopic control, to reduce the risk of complications. One patient gave a previous history of endometriosis, and two patients also had a history of very severe atypical pain. In these three patients, laparoscopy revealed endometriosis which was excised and confirmed on histological examination. In the remaining patient, laparoscopy was performed because a preoperative ultrasound scan had suggested the presence of an ovarian cyst; however, there was no evidence of this at laparoscopic examination.

Following the hysteroscopy, nine of the 20 patients were completely cured of pain and discharged. However, 11 patients still complained of pain and needed further treatment to control this. One patient, whom had been found to have endometriosis at laparoscopy, had a laparoscopic bilateral salpingo-oophorectomy which resulted in complete resolution of her symptoms. Nine of the patients had a hysterectomy either vaginally or laparoscopically; all patients were cured of pain following this procedure and subsequently discharged. Histology results in these patients revealed adenomyosis in three cases, endometriosis in one case and no abnormalities in the other five patients. One patient was put on mefenamic acid as an attempt to control her pain but then failed to attend for further follow-up as such the final outcome was unknown.

Analysis of the results by division of the patients into two groups dependent upon whether they suffered from pain prior to the MEA procedure or not (Table 1) shows in the group of women with no pelvic pain prior to the MEA procedure; $85.7 \%(6 / 7)$ of women were cured with hysteroscopic adhesiolysis alone. One of these seven patients had both hysteroscopic adhesiolysis and laparoscopic treatment of endometriosis; therefore, it is unclear which procedure cured her pain. However, in the group who complained of pain prior to the MEA procedure, only $10 \%(1 / 10)$ of women were cured with hysteroscopic adhesiolysis alone. Comparison of these two groups of

Table 1 Cure rates after hysteroscopy \pm adhesiolysis dependent upon presence of pain prior to MEA

\begin{tabular}{lll}
\hline & $\begin{array}{l}\text { Intrauterine adhesions } \\
\text { present }\end{array}$ & $\begin{array}{l}\text { Number of patients } \\
\text { cured (\%) }\end{array}$ \\
\hline Pain prior to & Yes 10 & $1(10)$ \\
MEA & No 2 & $1(50)^{\mathrm{a}}$ \\
No pain prior to & Yes 7 & $7(100)^{\mathrm{b}}$ \\
MEA & No 1 & $0(0)$ \\
\hline
\end{tabular}

\footnotetext{
${ }^{\text {a }}$ Patient cured by laparoscopic treatment of endometriosis at same time as hysteroscopy

${ }^{\mathrm{b}}$ One patient had laparoscopic treatment of endometriosis at same time as hysteroscopy
} 
patients using the Fisher's Exact test shows this difference is statistically significant $(P<0.01)$.

\section{Discussion}

From this study, the incidence of patients presenting to the gynaecological clinic with cyclical or intermittent pelvic pain following MEA is $11 \%$ with the time of presentation ranging from 3 to 41 months, which is comparable to those that have been found following the first generation ablation techniques [3-5]. Previous studies have concluded that pelvic pain post endometrial ablation occurs in women whom have previously been sterilised due to the development of haematosalpinges between the endometrial cavity and the blocked fallopian tube, which is known as postablation tubal sterilisation syndrome (PATSS) [6-8]. This has not been confirmed in our study as only $35 \%$ of our women had been sterilised prior to the MEA procedure. Therefore, the presumed pathogenesis in the remainder of our patients is occurrence of intrauterine adhesions possibly leading to the development of cornual or central haematometra which has been described previously after first generation endometrial ablations [9]. If this is the case, hysteroscopic adhesiolysis should, in theory, alleviate the pain in the majority of patients. In our study, $45 \%$ of all patients were cured with this procedure, and only $55 \%$ of patients needed to undergo major gynaecological surgery, which was mainly in the form of a hysterectomy.

In our study, $75 \%$ of women had a pelvic ultrasound performed preoperatively, but only $40 \%$ of these confirmed the presence of a haematometra. However, studies have shown that magnetic resonance imaging is probably superior at detecting small haematometra than ultrasound scans and that all scans are best done when the patient is symptomatic as the haematometra can resolve during the rest of the cycle [10]. More studies looking at preoperative diagnosis of these complications may help determine which group of patients may benefit from hysteroscopic adhesiolysis.

In our study, patients without pelvic pain prior to the MEA were statistically significantly more likely to be cured from hysteroscopic adhesiolysis than those whom complained of pain prior to the MEA, implying that they have only developed pain as a consequence of the MEA. This is supported by the fact that for five patients with pain preMEA who were not cured by hysteroscopic adhesiolysis, pathological study revealed two had endometriosis and three had adenomyosis, for which hysteroscopic adhesiolysis would not be the treatment of choice. Other similar studies confirm that the majority of patients undergoing hysterectomy, for continued bleeding or pain, following endometrial ablation have other pathology present such as adenomyosis or endometriosis $[2,11]$.

However, in our study in five patients who underwent hysterectomy, the pathology report showed no abnormality to account for the pain. It may be that our pathologists did not specifically look for evidence of cornual haematometra or PATSS as they were not aware of this complication. It has been suggested that pathologists need to be specifically asked to serially section the cornual areas of the uterus and the proximal fallopian tubes in these women to diagnose these conditions; however, they may still be missed if the patient is not symptomatic at the time of the hysterectomy [10].

Hysteroscopic adhesiolysis post MEA was found to be a safe procedure in this study as only two patients encountered complications, both of which were uterine perforations and neither case required extra treatment or developed further problems as a result.

This study is limited by the small number of cases included, but it is difficult to obtain large numbers of cases when the incidence is only $11 \%$. However, we are continuing to study this group of women to assess if the results continue to follow the same trend.

\section{Conclusion}

In conclusion, hysteroscopic adhesiolysis for patients presenting with pelvic pain post MEA seems to be a safe procedure. It appears to be most beneficial in the group of patients who do not give a history of pain prior to the MEA procedure, which in our study was almost half the patients and, as a consequence, avoided the need for major gynaecological surgery with its inherent risks and costs. All experiments comply with the current laws of the country in which they were performed.

\section{References}

1. National Institute of Clinical Excellence (2003) Guidance IPG7 Microwave endometrial ablation. National Institute of Clinical Excellence, London

2. Sharma B, Preston J, Ray C (2004) Microwave endometrial ablation for menorrhagia: outcome at 2 years-experience of a district general hospital. J Obstet Gynaecol 24(8):916-919

3. Jacobs SA, Blumenthal NJ (1994) Endometrial resection follow up: late onset of pain and the effect of depot medroxyprogesterone acetate. Br J Obstet Gynaecolo 101:605-609

4. Broadbent JAM, Magos AL (1995) Endometrial resection follow up: late onset of pain and the effect of depot medroxyprogesterone acetate [letter]. Br J Obstet Gynaecolo 102:587

5. Perrella RR, McLucas B (1994) Endometrial ablation post operative pain, sonographic findings, management and outcome. Minim Invasive Ther 3:317-321 
6. Townsend DE, McCausland V, McCausland A et al (1993) Postablation-tubal sterilisation syndrome. Obstet Gynecol 82(3):422424

7. Webb JC, Bush MR, Wood MD et al (1996) Hematosalpinx with pelvic pain after endometrial ablation confirms the postablation-tubal sterilization syndrome. J Am Assoc Gynecol Laparoscopists 3(3):419-421

8. Mall A, Shirk G, Van Voorhis BJ (2002) Previous tubal ligation is a risk factor for hysterectomy after rollerball endometrial ablation. Obstet Gynecol 100(4):659-664
9. McCausland AM, McCausland VM (2002) Frequency of symptomatic cornual haematometra and postablation tubal sterilization syndrome after total rollerball endometrial ablation: a ten year follow up. Am J Obstet Gynecol 186(6):1274-1280

10. McCausland AM, McCausland VM (2007) Long-term complications of endometrial ablation: Cause, diagnosis, treatment and prevention. J Minim Invasive Gynecol 14(4):399-406

11. Unger JB, Meeks GR (1996) Hysterectomy after endometrial ablation. Am J Obstet Gynecol 175(6):1432-1437 